\title{
Fixed-Time Complex Modified Function Projective Lag Synchronization of Chaotic (Hyperchaotic) Complex Systems
}

\author{
Xuan-Toa Tran and Hee-Jun Kang \\ School of Electrical Engineering, University of Ulsan, 93 Daehak-ro, Nam-gu, Ulsan 680-749, Republic of Korea \\ Correspondence should be addressed to Hee-Jun Kang; hjkang@ulsan.ac.kr
}

Received 21 February 2017; Revised 11 April 2017; Accepted 7 May 2017; Published 13 July 2017

Academic Editor: Sigurdur F. Hafstein

Copyright (c) 2017 Xuan-Toa Tran and Hee-Jun Kang. This is an open access article distributed under the Creative Commons Attribution License, which permits unrestricted use, distribution, and reproduction in any medium, provided the original work is properly cited.

\begin{abstract}
A novel fixed-time controller is proposed to perform fixed-time complex modified function projective lag synchronization of chaotic (hyperchaotic) complex systems. The synchronization is obtained after a finite time that could be preestablished without the knowledge of the initial states of both synchronized chaotic (hyperchaotic) complex systems. The global fixed-time stability of the closed-loop systems is rigorously proven based on Lyapunov analysis. Some simulation examples are provided to demonstrate the effectiveness and feasibility of the proposed method and verify the theoretical results.
\end{abstract}

\section{Introduction}

In the past two decades, much work has been devoted to investigate dynamic behavior and synchronization of chaotic (hyperchaotic) complex systems because of their potential applications. For instance, Gibbon and McGuinness [1] presented a complex set of Lorenz equations derived in laser optics and baroclinic instability, Zhang and Liu [2] applied synchronization to communication problem under considering time delay, and $\mathrm{Wu}$ et al. [3] proposed a method to improve the secure communications via passive synchronization of hyperchaotic complex systems. In the literature, researchers have introduced several types of synchronization of chaotic (hyperchaotic) complex systems such as complete synchronization (CoS) [4], antisynchronization (AS) [5], compound synchronization [6], projective synchronization (PS) [7], modified projective and modified function projective synchronization (MPS-MFPS) [8], combinationcombination synchronization [9], complete lag synchronization (CoLS) [10], general hybrid projective complete dislocated synchronization [11], modified projective lag synchronization (MPLS) [12], and modified function projective lag synchronization (MFPLS) [13]. Recently, some new types of synchronization that utilized complex scaling factors have been introduced to achieve the synchronization of both module and phase, the concept first proposed by Nian et al. [14]. Complex complete synchronization (CCoS) $[15,16]$, complex projective synchronization (CPS) [17], combination complex synchronization [18], complex modified projective synchronization (CMPS) [19], and complex function projective synchronization (CFPS) $[20,21]$ are some examples. In these synchronizations, the scaling factors are complex numbers (or functions) that increase the unpredictability and complexity; they have thus significant meaning for enhancing the security of communication [20]. Observation from [8, $20,21]$ shows that mentioned kinds of synchronizations only are special cases of complex modified function projective synchronization (CMFPS). Furthermore, there might be time delay between drive system and response system from practical point of view [13,20,21]; complex modified function projective lag synchronization (CMFPLS), which is a more general case, is rarely studied or not mentioned up to date.

In order to obtain such complex synchronization of chaotic (hyperchaotic) complex systems, some control methods such as Lyapunov-based control, feedback control and/or adaptive control [2, 5, 6, 8, 11-15, 17-20], passive control [3], finite-time stability theory-based control $[4,9,16]$, nonlinear observer-based control [7], backstepping-based control [10], and adaptive fuzzy logic control [21] have been proposed. In the mentioned methods, while the ones in $[4,9,16]$ ensure obtaining finite-time synchronization in which the convergence time still depends on both control design parameters 
and initial conditions, these remainders guarantee that the synchronization errors might converge to origin asymptotically in the best case. It is certain that synchronization errors could reach to zero in an amount of time which is more desirable object. Finite-time stability theory-based control methods have been known as useful and efficient techniques with some advanced properties such as fast and finitetime convergence, robustness, and high tracking precision [22]. In some last years, some researchers have introduced fixed-time stability concept, which is a further extension of finite-time stability one, in the literature [23-26]. The fixed-time stabilization problem of linear systems has solved in [23] based on nonlinear feedback control. Inspired by well-known super-twisting (STW) algorithm and high-order differentiator [27, 28], Basin et al. have proposed an adaptive STW-like controller with uniform finite/fixed convergence time in [24] and a continuous finite-time and fixed-time control method for an $n$-dimensional chain of integrators [25]. In [26], Polyakov et al. have introduced finite-time and fixed-time control methods for stabilizing linear multi-inputmultioutput systems. The fixed-time control methods ensure that the origin of the system is stabilized in some finite time that is not dependent on the initial conditions; in other words, the convergent time is completely predefined via designed controller parameters.

Motivated by the above discussion, the main goal of this article is to propose a novel fixed-time control law to, for the first time, obtain fixed-time complex modified function projective lag synchronization of chaotic (hyperchaotic) complex systems; that is, the modified function projective lag synchronization (CMFPLS) error states converge to zero in a predefined amount of time despite initial conditions. The global stability and fixed-time convergence are rigorously proven, and an upper bound of the convergence time is estimated.

The rest of this paper is organized into four sections. Section 2 gives some preliminaries and problem formulation. In Section 3, a fixed-time controller is introduced to solve the CMFPLS problem. Two illustrative examples are provided to demonstrate the effectiveness and the feasibility of the suggested control method in Section 4. Finally, some conclusions are drawn in Section 5.

Notations. $\mathbb{R}$ and $\mathbb{C}$ denote, respectively, the space of real number and complex number, and $\operatorname{diag}(\cdot)$ represents a blockdiagonal matrix. If $x \in \mathbb{R}$, the following notation is introduced for simplicity of expression [29]:

$$
x^{[\phi]}=|x|^{\phi} \operatorname{sign}(x),
$$

where $\phi>0$, and it can be verified that as $\phi \geq 1$,

$$
\frac{d}{d t} x^{[\phi]}=\phi|x|^{\phi-1} \dot{x} .
$$

If $x \in \mathbb{C}$, that is, $x=x^{r}+j x^{i}$ (superscripts $r$ and $i$ stand for the real and imaginary parts of the complex number $x$, and $j=\sqrt{-1})$, we then adopt the notation that $\bar{x}=x^{r}-j x^{i}, x^{[\phi]}=$ $\left(x^{r}\right)^{[\phi]}+j\left(x^{i}\right)^{[\phi]}, \mathbf{x}=\left(x_{1}, x_{2}, \ldots, x_{n}\right)^{\mathbf{T}} \in \mathbb{R}^{\mathbf{n}}, \mathbf{x}=\left(x_{1}, x_{2}, \ldots\right.$, $\left.x_{n}\right)^{\mathrm{T}} \in \mathbb{C}^{\mathbf{n}}$.

\section{Preliminaries and Problem Formulation}

First at all, some concepts related to finite-time stability and fixed-time stability given in [23] and a lemma that will be used in the analysis later are introduced as follows.

Consider the system

$$
\dot{\mathbf{x}}=\psi(t, \mathbf{x}), \quad \mathbf{x}(0)=\mathbf{x}_{0},
$$

where $\mathbf{x} \in \mathbb{R}^{n}$ and $\psi: \mathbb{R}_{+} \times \mathbb{R}^{n} \rightarrow \mathbb{R}^{n}$ is a nonlinear function which can be discontinuous. Assume the origin is an equilibrium point of (3).

Definition 1. The origin of (3) is said to be globally finitetime stable if it is globally asymptotically stable and any solution $\mathbf{x}\left(t, \mathbf{x}_{0}\right)$ of (3) reaches the equilibria at some finitetime moment; that is, $\mathbf{x}\left(t, \mathbf{x}_{0}\right)=0, \forall t \geq T\left(\mathbf{x}_{0}\right)$, where $T$ : $\mathbb{R}^{n} \rightarrow \mathbb{R}_{+} \cup\{0\}$ is the settling-time function.

Definition 2. The origin of (3) is said to be globally fixed-time stable if it is globally finite-time stable and the settling-time function $T\left(\mathbf{x}_{0}\right)$ is bounded; that is, $\exists T_{\max }>0: T\left(\mathbf{x}_{0}\right) \leq$ $T_{\max }, \forall \mathbf{x}_{0} \in \mathbb{R}^{n}$.

Lemma 3 (see [30], Jensen's inequality). One has

$$
\left(\sum_{i=1}^{m} z_{i}^{\theta_{2}}\right)^{1 / \theta_{2}} \leq\left(\sum_{i=1}^{m} z_{i}^{\theta_{1}}\right)^{1 / \theta_{1}}, \quad 0<\theta_{1}<\theta_{2}
$$

with $z_{i} \geq 0$ and $0 \leq i \leq m$.

Two different $n$-dimensional complex chaotic (hyperchaotic) systems under this study are described as follows.

The drive system is

$$
\dot{\mathbf{x}}(t)=\dot{\mathbf{x}}^{r}+j \dot{\mathbf{x}}^{i}=\mathbf{f}(\mathbf{x}(t), \overline{\mathbf{x}}(t), t),
$$

where $\mathbf{x}(t)$ and $\mathbf{f}(\mathbf{x}(t), \overline{\mathbf{x}}(t), t)$ are the state complex vector and the vector of nonlinear complex functions of the drive system, respectively.

And response system is

$$
\dot{\mathbf{y}}(t)=\dot{\mathbf{y}}^{r}+j \dot{\mathbf{y}}^{i}=\mathbf{g}(\mathbf{y}(t), \overline{\mathbf{y}}(t), t)+\mathbf{u}(t),
$$

where $\mathbf{y}(t), \mathbf{g}(\mathbf{y}(t), \overline{\mathbf{y}}(t), t)$, and $\mathbf{u}(t)$ are the state complex vector, the vector of nonlinear complex functions, and the control inputs of the controlled response system, respectively.

Define complex modified function projective lag synchronization (CMFPLS) error between the drive system and the controlled response system as

$$
\mathbf{e}(t)=\mathbf{e}^{r}+j \mathbf{e}^{i}=\mathbf{y}(t)-\boldsymbol{\Omega}(t) \mathbf{x}(t-\tau),
$$

where $\boldsymbol{\Omega}(t)=\boldsymbol{\Omega}^{r}(t)+j \boldsymbol{\Omega}^{i}(t)=\operatorname{diag}\left(\omega_{1}(t), \omega_{2}(t), \ldots, \omega_{n}(t)\right)$ is a function scaling matrix, $\omega_{k}(t)=\omega_{k}^{r}(t)+j \omega_{k}^{i}(t) \in \mathbb{C}$ are different and continuously differentiable bounded functions, $\omega_{k}(t) \neq 0$ for all $t \geq 0, \tau$ is positive time lag, and $\mathbf{e}(t)=$ $\left(e_{1}(t), e_{2}(t), \ldots, e_{n}(t)\right)^{T}$; here $e_{k}(t)=e_{k}^{r}(t)+j e_{k}^{i}(t), \quad(k=$ $1,2, \ldots, n)$. For convenience of notation, we utilize $e_{k}, e_{k}^{r}$, and $e_{k}^{i}$ instead of $e_{k}, e_{k}^{r}(t)$, and $e_{k}^{i}(t)$, respectively. 
TABLE 1: Some types of synchronization.

\begin{tabular}{lcc}
\hline Function scaling matrix & \multicolumn{2}{c}{ Type of synchronization } \\
$\boldsymbol{\Omega}(t)=\boldsymbol{\Omega}^{r}(t)+j \boldsymbol{\Omega}^{i}(t)=\operatorname{diag}\left(\omega_{1}(t), \omega_{2}(t), \ldots, \omega_{n}(t)\right)$ & $\tau \neq 0$ & $\tau=0$ \\
$\omega_{k}(t)=\omega_{k}^{r}(t)+j \omega_{k}^{i}(t),(k=1,2, \ldots, n)$ & CFPLS & CFPS \\
\hline$\omega_{k}(t),(k=1,2, \ldots, n)$ are identical complex functions & CMPLS & CMPS \\
$\omega_{k}(t),(k=1,2, \ldots, n)$ are different complex constants & CPLS & CPS \\
$\omega_{k}(t),(k=1,2, \ldots, n)$ are identical complex constants & CCoLS & CCoS \\
$\omega_{k}(t)=1+j,(k=1,2, \ldots, n)$ & MFPLS & CAS \\
$\omega_{k}(t)=-1-j,(k=1,2, \ldots, n)$ & FPLS \\
$\omega_{k}^{r}(t),(k=1,2, \ldots, n)$ are different real functions and $\boldsymbol{\Omega}^{i}(t)=0$ & MPLS & MFPS \\
$\omega_{k}^{r}(t),(k=1,2, \ldots, n)$ are identical real functions and $\boldsymbol{\Omega}^{i}(t)=0$ & PLS & FPS \\
$\omega_{k}^{r}(t),(k=1,2, \ldots, n)$ are different real constants and $\boldsymbol{\Omega}^{i}(t)=0$ & CoLS \\
$\omega_{k}^{r}(t),(k=1,2, \ldots, n)$ are identical real constants and $\boldsymbol{\Omega}^{i}(t)=0$ & MAS \\
$\omega_{k}(t)=1,(k=1,2, \ldots, n)$ & PS \\
$\omega_{k}(t)=-1,(k=1,2, \ldots, n)$ & CoS \\
\hline
\end{tabular}

Then, the CMFPLS error dynamics is determined by the following equation:

$$
\begin{aligned}
\dot{e}_{k}= & \dot{e}_{k}^{r}+j \dot{e}_{k}^{i} \\
= & -\omega_{k}(t) f_{k}(\mathbf{x}(t-\tau), \overline{\mathbf{x}}(t-\tau), t-\tau) \\
& -\dot{\omega}_{k}(t) x_{k}(t-\tau)+g_{k}(\mathbf{y}(t), \overline{\mathbf{y}}(t), t)+u_{k}(t) .
\end{aligned}
$$

Definition 4. The fixed-time complex modified function projective lag synchronization between the drive system (3) and the response system (4) is achieved if there exists a time moment $T>0$, which is independent of initial error states, $\mathbf{e}\left(t_{0}\right)$, such that $\|\mathbf{e}(t)\|=0, \forall t \geq t_{0}+T$.

The main goal of this article is to design control laws $u_{k}(t),(k=1,2, \ldots, n)$ such that the resulting closed-loop system is global fixed-time stability; that is, the fixed-time CMFPLS of the error system (5) is obtained in the sense of Definition 4.

Remark 5. The differential equation of form (3) is the expression of most of the well-known chaotic (hyperchaotic) complex systems such as chaotic (hyperchaotic) complex Chen, Lorenz, and Lü systems.

Remark 6. Besides several kinds of synchronization mentioned in the Introduction, CMFPLS considered in this study also consists of some other special cases such as complex function projective lag synchronization (CFPLS), complex modified projective lag synchronization (CMPLS), complex projective lag synchronization (CPLS), complex complete lag synchronization (CCoLS), complex lag antisynchronization (CLAS), complex antisynchronization (CAS), function projective lag synchronization (FPLS), function projective synchronization (PLS), projective lag synchronization (PLS), and lag antisynchronization (LAS) summarized in Table 1.

\section{Main Results}

In this section, a novel control method is proposed to achieve the fixed-time CMFPLS between the drive system (3) and the controlled response system (4). The main result of the article is summarized in the following theorem.

Theorem 7. Consider the CMFPLS error dynamics (8). If a feedback control law is designed as

$$
\begin{aligned}
u_{k}(t)= & u_{k}^{r}+j u_{k}^{i} \\
= & \omega_{k}(t) f_{k}(\mathbf{x}(t-\tau), \overline{\mathbf{x}}(t-\tau), t-\tau) \\
& +\dot{\omega}_{k}(t) x_{k}(t-\tau)-g_{k}(\mathbf{y}(t), \overline{\mathbf{y}}(t), t) \\
& -h_{1 k} e_{k}^{\left[2-\beta_{k}\right]}-h_{2 k}\left([\mathfrak{e}]^{\lambda_{k}\left|e_{k}^{r}\right|} e_{k}^{r}+j[\mathfrak{e}]^{\lambda_{k}\left|e_{k}^{i}\right|} e_{k}^{i}\right) \\
& -h_{3 k} e_{k}^{\left[\beta_{k}\right]},
\end{aligned}
$$

where $h_{1 k}, h_{2 k}$, and $h_{3 k}$ are positive constants and satisfy $4 h_{1 k} h_{3 k}>h_{2 k}^{2}, 0<\beta_{k}<1, \lambda_{k}>0,(k=1,2, \ldots, n)$, and $[\mathrm{e}]$ is Euler's number, then the origin of the resulting closedloop system is globally fixed-time stable; that is, the fixedtime CMFPLS between the drive system (3) and the controlled response system (4) is obtained in the sense of Definition 4. In addition, the CMFPLS error states of (8) reach to zero at most after the fixed time

$$
\begin{gathered}
T=\frac{2}{\left(1-\beta_{k}\right) \sqrt{4 h_{1 k} h_{3 k}-h_{2 k}^{2}}}\left(\frac{\pi}{2}\right. \\
\left.-\tan ^{-1} \frac{h_{2 k}}{\sqrt{4 h_{1 k} h_{3 k}-h_{2 k}^{2}}}\right) .
\end{gathered}
$$

Proof. The CMFPLS error dynamics (8) under the control law (9) results in the closed-loop system represented in the form

$$
\begin{aligned}
\dot{e}_{k}= & \dot{e}_{k}^{r}+j \dot{e}_{k}^{i} \\
= & -h_{1 k} e_{k}^{\left[2-\beta_{k}\right]}-h_{2 k}\left([\mathfrak{e}]^{\lambda_{k}\left|e_{k}^{r}\right|} e_{k}^{r}+j[\mathfrak{e}]^{\lambda_{k}\left|e_{k}^{i}\right|} e_{k}^{i}\right) \\
& -h_{3 k} e_{k}^{\left[\beta_{k}\right]} .
\end{aligned}
$$


The resulting closed-loop system can be rewritten as

$$
\begin{aligned}
& \dot{e}_{k}^{r}=-h_{1 k}\left(e_{k}^{r}\right)^{\left[2-\beta_{k}\right]}-h_{2 k}[\mathfrak{e}]^{\lambda_{k}\left|e_{k}^{r}\right|} e_{k}^{r}-h_{3 k}\left(e_{k}^{r}\right)^{\left[\beta_{k}\right]}, \\
& \dot{e}_{k}^{i}=-h_{1 k}\left(e_{k}^{i}\right)^{\left[2-\beta_{k}\right]}-h_{2 k}[\mathfrak{e}]^{\lambda_{k}\left|e_{k}^{i}\right|} e_{k}^{i}-h_{3 k}\left(e_{k}^{i}\right)^{\left[\beta_{k}\right]} .
\end{aligned}
$$

Consider the following Lyapunov function candidate

$$
V=\sum_{k=1}^{n}\left[\left(e_{k}^{r}\right)^{2}+\left(e_{k}^{i}\right)^{2}\right] \text {. }
$$

Its time derivatives along the trajectories of (12) lead to

$$
\begin{aligned}
\dot{V} & =2 \sum_{k=1}^{n} e_{k}^{r}\left[-h_{1 k}\left(e_{k}^{r}\right)^{\left[2-\beta_{k}\right]}-h_{2 k}[e]^{\lambda_{k}\left|e_{k}^{r}\right|} e_{k}^{r}\right. \\
& \left.-h_{3 k}\left(e_{k}^{r}\right)^{\left[\beta_{k}\right]}\right]+2 \sum_{k=1}^{n} e_{k}^{i}\left[-h_{1 k}\left(e_{k}^{i}\right)^{\left[2-\beta_{k}\right]}\right. \\
& \left.-h_{2 k}[e]^{\lambda_{k}\left|e_{k}^{i}\right|} e_{k}^{i}-h_{3 k}\left(e_{k}^{i}\right)^{\left[\beta_{k}\right]}\right] \\
& =2 \sum_{k=1}^{n}\left(-h_{1 k}\left|e_{k}^{r}\right|^{3-\beta_{k}}-h_{2 k}[e]^{\lambda_{k}\left|e_{k}^{r}\right|}\left(e_{k}^{r}\right)^{2}\right. \\
& \left.-h_{3 k}\left|e_{k}^{r}\right|^{1+\beta_{k}}\right)+2 \sum_{k=1}^{n}\left(-h_{1 k}\left|e_{k}^{i}\right|^{3-\beta_{k}}\right. \\
& \left.-h_{2 k}[\mathfrak{e}]^{\lambda_{k}\left|e_{k}^{i}\right|}\left(e_{k}^{i}\right)^{2}-h_{3 k}\left|e_{k}^{i}\right|^{1+\beta_{k}}\right) .
\end{aligned}
$$

Applying Lemma 3, we can have

$$
\begin{aligned}
\dot{V} & \leq 2 \sum_{k=1}^{n}\left\{-h_{1 k}\left[\left(e_{k}^{r}\right)^{2}+\left(e_{k}^{i}\right)^{2}\right]^{\left(3-\beta_{k}\right) / 2}\right. \\
& -h_{2 k}\left[\left(e_{k}^{r}\right)^{2}+\left(e_{k}^{i}\right)^{2}\right] \\
& \left.-h_{3 k}\left[\left(e_{k}^{r}\right)^{2}+\left(e_{k}^{i}\right)^{2}\right]^{\left(1+\beta_{k}\right) / 2}\right\} \leq-2 h_{1 k} V^{\left(3-\beta_{k}\right) / 2} \\
& -2 h_{2 k} V-2 h_{3 k} V^{\left(1+\beta_{k}\right) / 2} .
\end{aligned}
$$

Hence, the Lyapunov theorem [31] indicates that origin of (12) is globally asymptotically stable. Further, we will show that the convergence takes place in finite time.

We consider the differential equation

$$
\begin{aligned}
& \dot{\varphi}=-2 h_{1 k} \varphi^{\left(3-\beta_{k}\right) / 2}-2 h_{2 k} \varphi-2 h_{3 k} \varphi^{\left(1+\beta_{k}\right) / 2}, \\
& \varphi(0)=\varphi_{0} \geq 0 .
\end{aligned}
$$

It follows that

$$
\begin{aligned}
d t & =-\frac{d \varphi}{2\left(h_{1 k} \varphi^{1-\beta}+h_{2 k} \varphi^{\left(1-\beta_{k}\right) / 2}+h_{3 k}\right) \varphi^{\left(\beta_{k}+1\right) / 2}} \\
& =-\frac{1}{1-\beta_{k}} \frac{d \varphi^{\left(1-\beta_{k}\right) / 2}}{\left(h_{1 k} \varphi^{1-\beta_{k}}+h_{2 k} \varphi^{\left(1-\beta_{k}\right) / 2}+h_{3 k}\right)} .
\end{aligned}
$$

The solution of (17) found under the condition, $h_{2 k}^{2} \leq 4 h_{1 k} h_{3 k}$, satisfies the following equation:

$$
\begin{aligned}
t= & \frac{2}{\left(1-\beta_{k}\right) \sqrt{4 h_{1 k} h_{3 k}-h_{2 k}^{2}}}\left(\tan ^{-1} \frac{2 h_{1 k} \varphi(0)+h_{2 k}}{\sqrt{4 h_{1 k} h_{3 k}-h_{2 k}^{2}}}\right. \\
& \left.-\tan ^{-1} \frac{2 h_{1 k} \varphi(t)+h_{2 k}}{\sqrt{4 h_{1 k} h_{3 k}-h_{2 k}^{2}}}\right) .
\end{aligned}
$$

Since expression (16) implies that $\varphi(t)$ is decreasing, (18) indicates that $\varphi(t)$ will become zero in finite time.

$$
\begin{aligned}
& T_{\varphi} \\
& =\frac{2}{\left(1-\beta_{k}\right) \sqrt{4 h_{1 k} h_{3 k}-h_{2 k}^{2}}}\left(\tan ^{-1} \frac{2 h_{1 k} \varphi(0)+h_{2 k}}{\sqrt{4 h_{1 k} h_{3 k}-h_{2 k}^{2}}}\right. \\
& \left.-\tan ^{-1} \frac{h_{2 k}}{\sqrt{4 h_{1 k} h_{3 k}-h_{2 k}^{2}}}\right) .
\end{aligned}
$$

In addition, on the basis of the comparison principle [32], we can obtain that $V(t) \leq \varphi(t)$ when $V(t=0) \leq \varphi_{0}$, and it follows that $V(t)$, and therefore the origin of (12), converge to zero in finite time $T_{V} \leq T_{\varphi}$, with

$T_{V}$

$$
\begin{aligned}
& =\frac{2}{\left(1-\beta_{k}\right) \sqrt{4 h_{1 k} h_{3 k}-h_{2 k}^{2}}}\left(\tan ^{-1} \frac{2 h_{1 k} V(t=0)+h_{2 k}}{\sqrt{4 h_{1 k} h_{3 k}-h_{2 k}^{2}}}\right. \\
& \left.-\tan ^{-1} \frac{h_{2 k}}{\sqrt{4 h_{1 k} h_{3 k}-h_{2 k}^{2}}}\right) .
\end{aligned}
$$

Thus, the origin of (12) is globally finite-time stable on the basic of Definition 1 .

It can be easy to show that $T_{\varphi}$ is bounded by

$$
\begin{gathered}
T_{V} \leq T=\frac{2}{\left(1-\beta_{k}\right) \sqrt{4 h_{1 k} h_{3 k}-h_{2 k}^{2}}}\left(\frac{\pi}{2}\right. \\
\left.-\tan ^{-1} \frac{h_{2 k}}{\sqrt{4 h_{1 k} h_{3 k}-h_{2 k}^{2}}}\right) .
\end{gathered}
$$

Since the above expression of $T$ is only dependent on the controller parameters, it follows from Definition 2 that the origin of (12) is globally fixed-time stable. Consequently, the fixed-time CMFPLS between the drive system (3) and the controlled response system (4) is achieved in the sense of Definition 4. Theorem 7 is proven. 

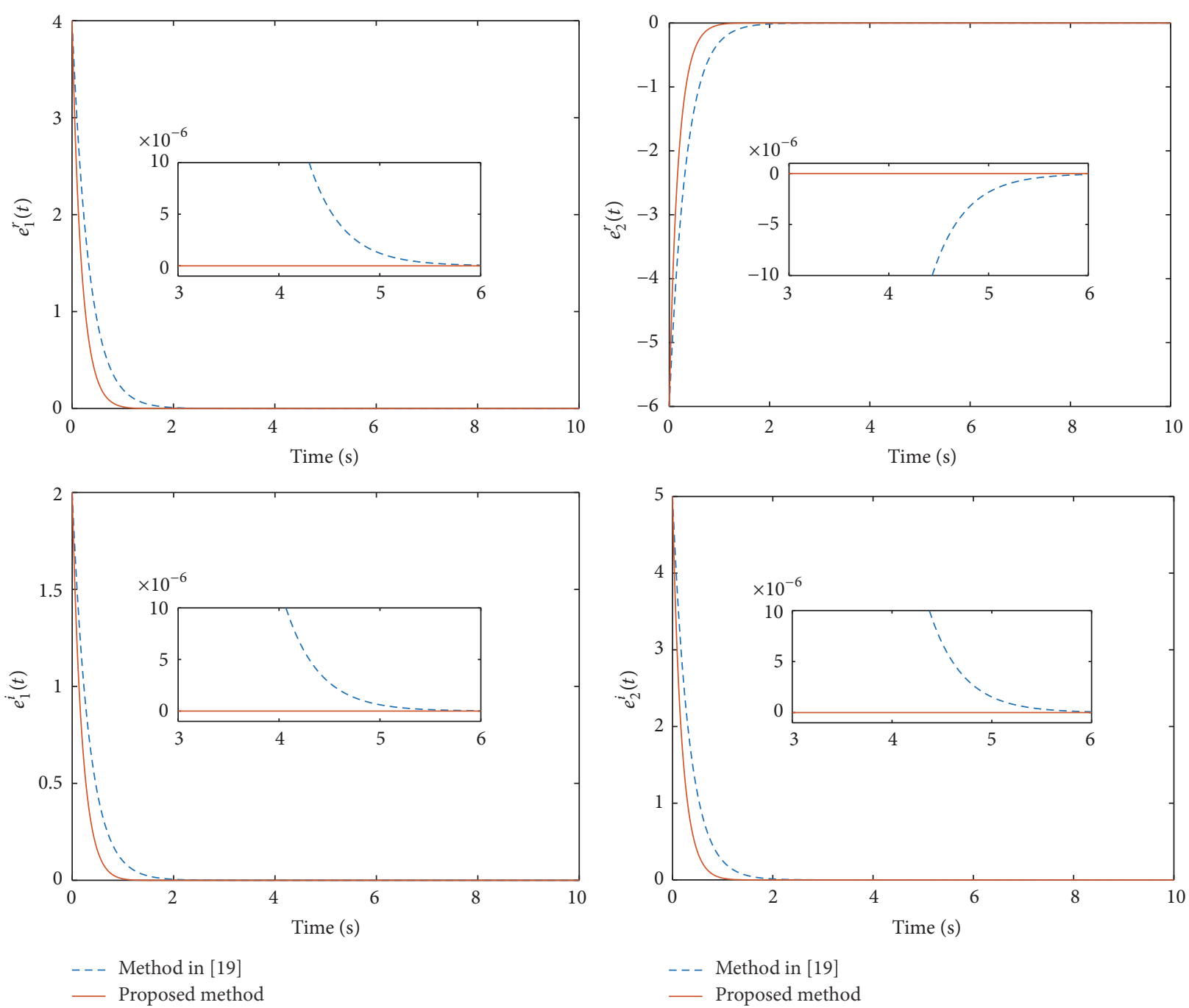

FIgURE 1: The time histories of the CMPS error states.

Remark 8. Regarding differential equation (11), $\mid-h_{1 k} e_{k}^{\left[2-\beta_{k}\right]}-$ $h_{2 k}\left([\mathfrak{e}]^{\lambda_{k}\left|e_{k}^{r}\right|} e_{k}^{r}+j[\mathfrak{e}]^{\lambda_{k}\left|e_{k}^{i}\right|} e_{k}^{i}\right) \mid$ holds a dominant function that ensures fast convergence speed while $\left|e_{k}\right|$ is much larger than 1 , and $h_{3 k} e_{k}^{\left[\beta_{k}\right]}$ takes the dominant one that determines finitetime convergence while $\left|e_{k}\right|$ is much smaller than 1 . Moreover, the "soft gain" $h_{2 \kappa}[\mathfrak{e}]^{\lambda_{k}\left|e_{k}^{r}\right|}$ (or $h_{2 \kappa}[\mathfrak{e}]^{\lambda_{k}\left|e_{k}^{i}\right|}$ ) is small if $\left|e_{k}^{r}\right|$ (or $\left.\left|e_{k}^{i}\right|\right)$ is close to zero. It implies that the influence of $h_{3 k} e_{k}^{\left[\beta_{k}\right]}$ is strong in the finite-time convergence period.

\section{Numerical Simulations}

Two illustrative examples are provided to demonstrate the effectiveness and applicability of the proposed control method in this section. In the numerical simulations, the fourth-order Runge-Kutta method is used to solve differential equations with a time step size of 0.001 .

4.1. Example 1. This example demonstrates the efficacy of the proposed approach by comparing to the existing method [19] through the CMPS between two chaotic systems. It should be noted that chaotic complex systems and the controller parameters of the existing method were directly taken from [19] to make a fair comparison.

The chaotic complex Lorenz system is assumed as the drive system given by

$$
\begin{aligned}
& \dot{x}_{1}=14\left(x_{2}-x_{1}\right), \\
& \dot{x}_{2}=35 x_{1}-x_{2}-x_{1} x_{3}, \\
& \dot{x}_{3}=\frac{1}{2}\left(\bar{x}_{1} x_{2}+x_{1} \bar{x}_{2}\right)-3.7 x_{3}
\end{aligned}
$$

and the chaotic complex Lu system is used as the controlled response system as

$$
\begin{aligned}
& \dot{y}_{1}=40\left(y_{2}-y_{1}\right), \\
& \dot{y}_{2}=22 y_{2}-y_{1} y_{3}, \\
& \dot{y}_{3}=\frac{1}{2}\left(\bar{y}_{1} y_{2}+y_{1} \bar{y}_{2}\right)-5 y_{3} .
\end{aligned}
$$



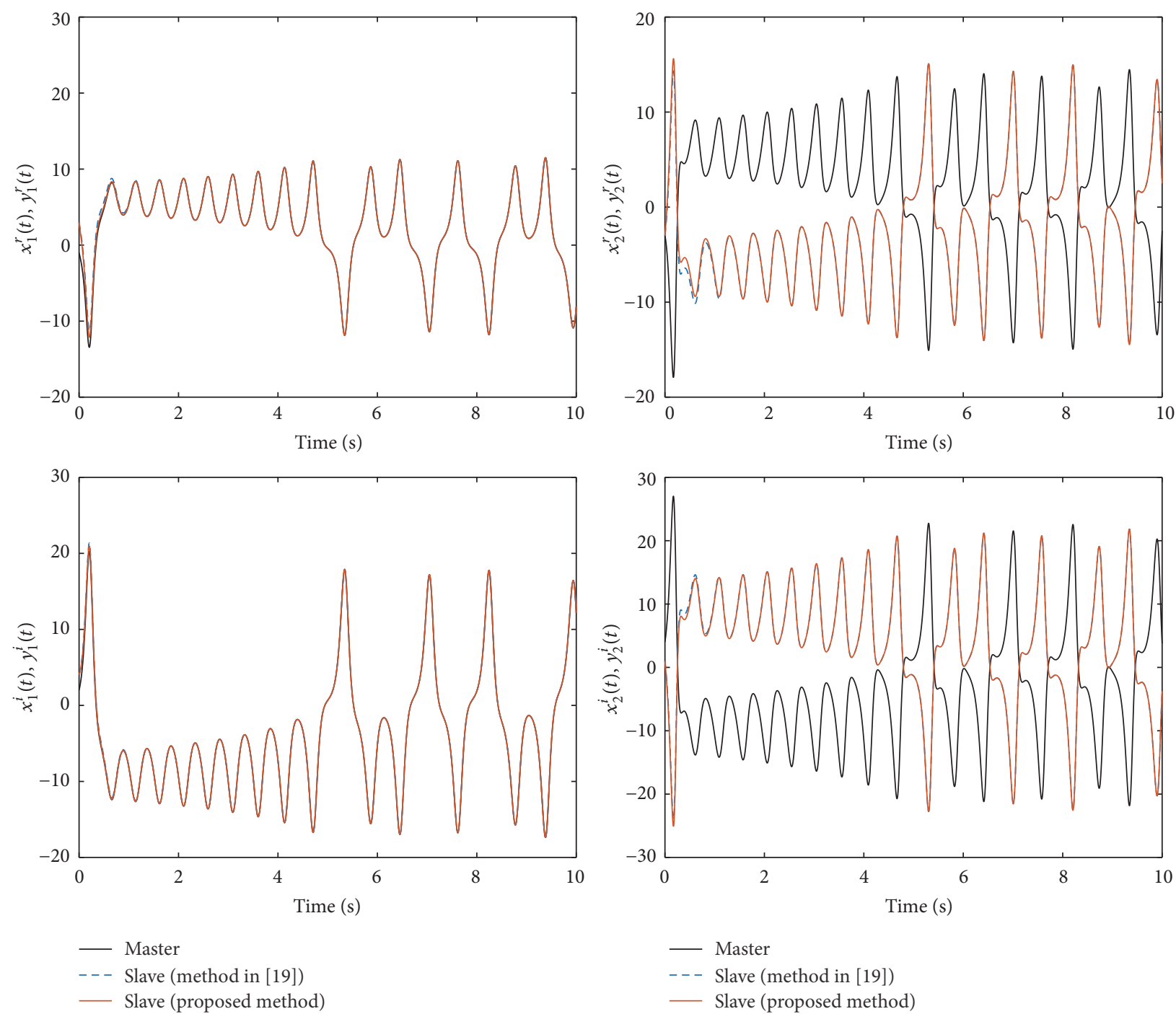

FIGURE 2: The time histories of the state variables of the drive system (22) and the controlled response system (23).

The initial states of systems (22) and (23) are chosen as $\mathbf{x}(0)=$ $[-1+j 2,-3+j 4,5]^{T}$ and $\mathbf{y}(0)=[3+j 4,-3+j, 3]^{T}$, respectively. Based on Remark 6 and Table 1, the CMPS is obtained by choosing the time delay as $\tau=0$ and the factor scaling functions as $\Omega=\operatorname{diag}(1,-1,0)$.

The proposed control law is designed as (9) where the parameter values are set as $h_{1 k}=2, h_{2 k}=1, h_{3 k}=2, \lambda_{k}=$ $0.1, \beta_{k}=0.8,(k=1,2,3)$, and then the upper bound of the convergence time is $T_{1} \approx 3.4 \mathrm{~s}$ based on (10), which is not dependent on the initial conditions of the chaotic systems.

The numerical simulation results are depicted in Figures 1 and 2. It is observed that all the CMPS errors reach origin in a finite amount of time that is less than $T_{1}=3.4 \mathrm{~s}$, which implies that the fixed-time CMPS between the drive system (22) and controlled response system (23) is achieved as in Figure 2. In contrast, we can see that the CMPS errors under the method in [19] is to converge to zero asymptotically; that is, $\lim _{t \rightarrow+\infty}\left|e_{i}\right|=0$. Furthermore, we could conclude from Figure 1 that the proposed method exhibits much better performance than the method in [19] does.
4.2. Example 2. The CMFPLS between two hyperchaotic complex systems is investigated in this example to completely verify the theoretical result introduced. The hyperchaotic complex Lu system [12] and hyperchaotic complex Lorenz system [12] are adopted as the drive system and the controlled response system, respectively.

The drive system is described as follows:

$$
\begin{aligned}
& x_{1}=42\left(x_{2}-x_{1}\right)+x_{4}, \\
& x_{2}=25 x_{2}-x_{1} x_{3}+x_{4}, \\
& x_{3}=\frac{1}{2}\left(\bar{x}_{1} x_{2}+x_{1} \bar{x}_{2}\right)-6 x_{3}, \\
& x_{4}=\frac{1}{2}\left(\bar{x}_{1} x_{2}+x_{1} \bar{x}_{2}\right)-5 x_{4}
\end{aligned}
$$

and the controlled response system is expressed as

$$
\begin{aligned}
& y_{1}=14\left(y_{2}-y_{1}\right)+j y_{4}, \\
& y_{2}=40 y_{1}-y_{2}-y_{1} y_{3}+j y_{4},
\end{aligned}
$$



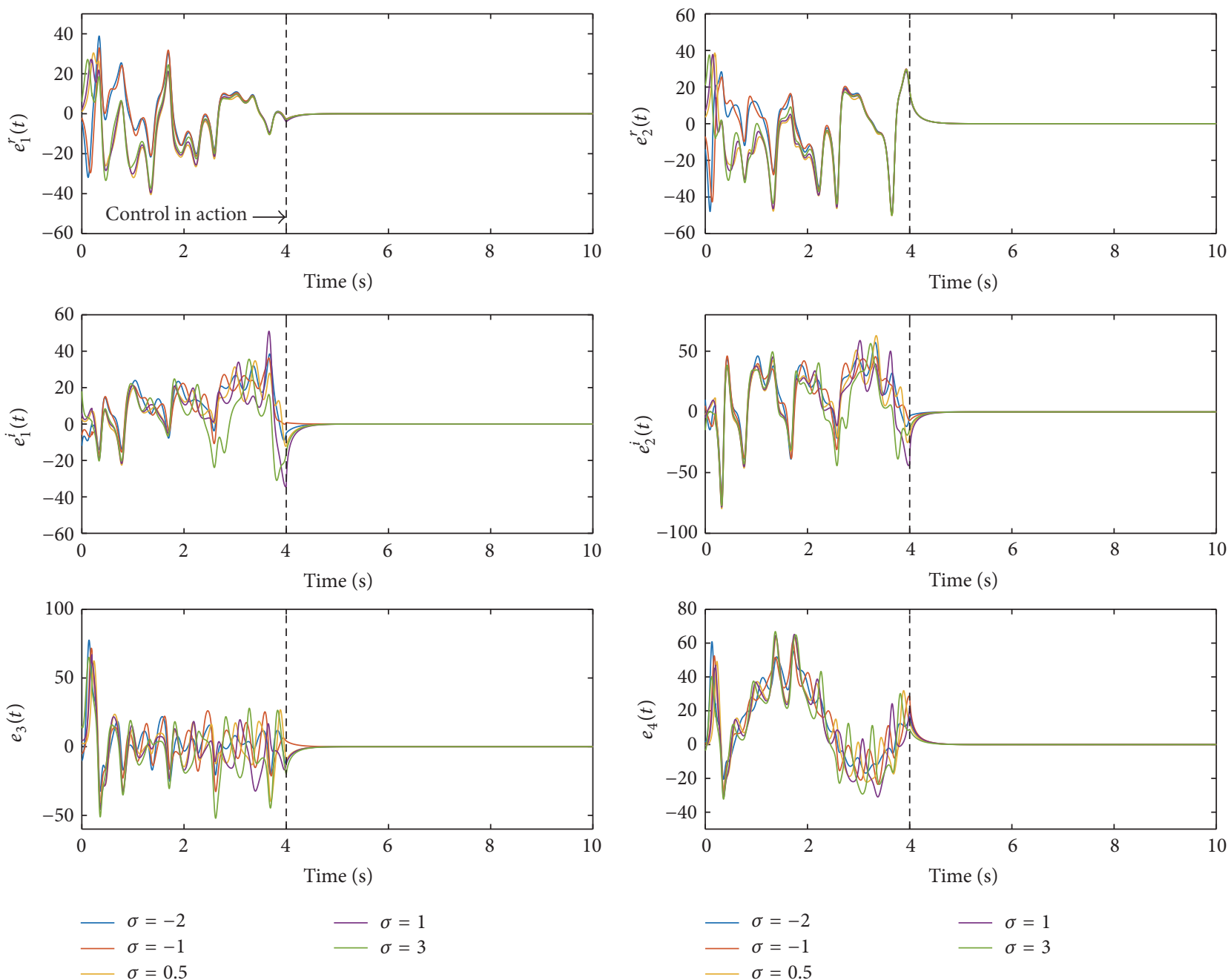

Figure 3: The time histories of the CMFPLS error states.

$$
\begin{aligned}
& y_{3}=\frac{1}{2}\left(\bar{y}_{1} y_{2}+y_{1} \bar{y}_{2}\right)-5 y_{3}, \\
& y_{4}=\frac{1}{2}\left(\bar{y}_{1} y_{2}+y_{1} \bar{y}_{2}\right)-13 y_{4} .
\end{aligned}
$$

To show that the upper bound of the convergence time, $T_{2}$, is independent of the initial conditions of synchronized systems, we will consider five cases in this example. The initial condition of the drive system (24) is set as $\mathbf{x}(0)=[-1+$ $j 2,-2+j 3,4,-3]^{T}$, and the initial condition of the controlled response system $(25)$ is selected in the form of $\mathbf{y}(0)=\sigma *[2+$ $j 6,7-j 5,5,-1]^{T}$, and here $\sigma=\{-2,-1,0.5,1,3\}$. The time delay is chosen as $\tau=0.2$, and the scaling factor functions are chosen as $\omega_{1}(t)=1+j \sin (t), \omega_{2}(t)=2-j \cos (t), \omega_{3}(t)=$ 1.5, $\omega_{4}(t)=\cos (2 t)$.

For this example, the controller parameters are $h_{1 k}=$ $2, h_{2 k}=1, h_{3 k}=2, \lambda_{k}=0.1$, and $\beta_{k}=0.65,(k=1,2,3,4)$; then this yields $T_{2}=1.94 \mathrm{~s}$ from (10). It is assumed that the controller is activated at $t_{0}=3 \mathrm{~s}$, and the upper bound of the whole convergence time, thus, is $t_{0}+T_{2}=5.94 \mathrm{~s}$.
The time responses of the CMFPLS error states are displayed in Figure 3. All the synchronization errors reach zero within the period $t_{0}+T_{2}=5.94 \mathrm{~s}$ despite the different initial conditions; evidently, the CMFPLS could be obtained in fixed time.

\section{Conclusions}

In this work, a novel fixed-time control method has been proposed to achieve fixed-time complex modified function projective lag synchronization of chaotic (hyperchaotic) complex systems. The numerical simulations have been given to verify the theoretical results and demonstrate the effectiveness and feasibility of the suggested scheme. It should be noted that the proposed method could be extended to either chaos control problems or synchronization types of chaotic (hyperchaotic) systems with different orders and non-square scaling factor matrices.

\section{Conflicts of Interest}

The authors declare that there are no conflicts of interest regarding the publication of this paper. 


\section{Acknowledgments}

This research was supported by Basic Science Research Program through the National Research Foundation of Korea (NRF) funded by the Ministry of Education (NRF2016R1D1A3B03930496).

\section{References}

[1] J. D. Gibbon and M. J. McGuinness, "The real and complex Lorenz equations in rotating fluids and lasers," Physica $D$. Nonlinear Phenomena, vol. 5, no. 1, pp. 108-122, 1982.

[2] F. Zhang and S. Liu, "Self-time-delay synchronization of timedelay coupled complex chaotic system and its applications to communication," International Journal of Modern Physics C. Computational Physics and Physical Computation, vol. 25, no. 3, Article ID 1350102, 1350102, 13 pages, 2014.

[3] X. Wu, C. Zhu, and H. Kan, "An improved secure communication scheme based passive synchronization of hyperchaotic complex nonlinear system," Applied Mathematics and Computation, vol. 252, pp. 201-214, 2015.

[4] X. B. Zhou, M. R. Jiang, and X. M. Cai, "Synchronization of a novel hyperchaotic complex-variable system based on finitetime stability theory," Entropy, vol. 15, no. 10, pp. 4334-4344, 2013.

[5] J. Sun and Y. Shen, "Adaptive anti-synchronization of chaotic complex systems and chaotic real systems with unknown parameters," Journal of Vibration and Control, vol. 22, no. 13, pp. 2992-3003, 2016.

[6] J. Sun, Y. Shen, Q. Yin, and C. Xu, "Compound synchronization of four memristor chaotic oscillator systems and secure communication," Chaos. An Interdisciplinary Journal of Nonlinear Science, vol. 23, no. 1, Article ID 013140, 013140, 10 pages, 2013.

[7] P. Liu, H. J. Song, and X. Li, "Observe-based projective synchronization of chaotic complex modified van der pol-duffing oscillator with application to secure communication," Journal of Computational and Nonlinear Dynamics, vol. 10, no. 5, Article ID 051015, 2015.

[8] J. Sun, Y. Shen, and X. Zhang, "Modified projective and modified function projective synchronization of a class of real nonlinear systems and a class of complex nonlinear systems," Nonlinear Dynamics. An International Journal of Nonlinear Dynamics and Chaos in Engineering Systems, vol. 78, no. 3, pp. 1755-1764, 2014.

[9] J. Sun, Y. Shen, X. Wang, and J. Chen, "Finite-time combinationcombination synchronization of four different chaotic systems with unknown parameters via sliding mode control," Nonlinear Dynamics. An International Journal of Nonlinear Dynamics and Chaos in Engineering Systems, vol. 76, no. 1, pp. 383-397, 2014.

[10] X. Wang and H. Zhang, "Backstepping-based lag synchronization of a complex permanent magnet synchronous motor system," Chinese Physics B, vol. 22, no. 4, Article ID 048902, 2013.

[11] J. Sun, Y. Wang, L. Yao, Y. Shen, and G. Cui, "General hybrid projective complete dislocated synchronization between a class of chaotic real nonlinear systems and a class of chaotic complex nonlinear systems," Applied Mathematical Modelling. Simulation and Computation for Engineering and Environmental Systems, vol. 39, no. 20, pp. 6150-6164, 2015.

[12] G. M. Mahmoud and E. E. Mahmoud, "Modified projective lag synchronization of two nonidentical hyperchaotic complex nonlinear systems," International Journal of Bifurcation and Chaos, vol. 21, no. 8, pp. 2369-2379, 2011.

[13] X. Wang and N. Wei, "Modified function projective lag synchronization of hyperchaotic complex systems with parameter perturbations and external perturbations," Journal of Vibration and Control, vol. 21, no. 16, pp. 3266-3280, 2015.

[14] F. Nian, X. Wang, Y. Niu, and D. Lin, "Module-phase synchronization in complex dynamic system," Applied Mathematics and Computation, vol. 217, no. 6, pp. 2481-2489, 2010.

[15] E. E. Mahmoud, "Complex complete synchronization of two nonidentical hyperchaotic complex nonlinear systems," Mathematical Methods in the Applied Sciences, vol. 37, no. 3, pp. 321328, 2014.

[16] J. Sun, Y. Wang, Y. Wang, and Y. Shen, "Finite-time synchronization between two complex-variable chaotic systems with unknown parameters via nonsingular terminal sliding mode control," Nonlinear Dynamics. An International Journal of Nonlinear Dynamics and Chaos in Engineering Systems, vol. 85, no. 2, pp. 1105-1117, 2016.

[17] Z. Wu, J. Duan, and X. Fu, "Complex projective synchronization in coupled chaotic complex dynamical systems," Nonlinear Dynamics. An International Journal of Nonlinear Dynamics and Chaos in Engineering Systems, vol. 69, no. 3, pp. 771-779, 2012.

[18] J. Sun, G. Cui, Y. Wang, and Y. Shen, "Combination complex synchronization of three chaotic complex systems," Nonlinear Dynamics. An International Journal of Nonlinear Dynamics and Chaos in Engineering Systems, vol. 79, no. 2, pp. 953-965, 2015.

[19] G. M. Mahmoud and E. E. Mahmoud, "Complex modified projective synchronization of two chaotic complex nonlinear systems," Nonlinear Dynamics. An International Journal of Nonlinear Dynamics and Chaos in Engineering Systems, vol. 73, no. 4, pp. 2231-2240, 2013.

[20] F. Zhang and S. Liu, "Adaptive complex function projective synchronization of uncertain complex chaotic systems," Journal of Computational and Nonlinear Dynamics, vol. 11, no. 1, Article ID 011013, 2016.

[21] J. W. Lee, S. M. Lee, and S. C. Won, "Complex function projective synchronization of general networked chaotic systems by using complex adaptive fuzzy logic," Nonlinear Dynamics. An International Journal of Nonlinear Dynamics and Chaos in Engineering Systems, vol. 81, no. 4, pp. 2095-2106, 2015.

[22] S. P. Bhat and D. S. Bernstein, "Finite-time stability of continuous autonomous systems," SIAM Journal on Control and Optimization, vol. 38, no. 3, pp. 751-766, 2000.

[23] A. Polyakov, "Nonlinear feedback design for fixed-time stabilization of linear control systems," Institute of Electrical and Electronics Engineers. Transactions on Automatic Control, vol. 57, no. 8, pp. 2106-2110, 2012.

[24] M. Basin, C. B. Panathula, and Y. Shtessel, "Adaptive uniform finite-/fixed-time convergent second-order sliding-mode control," International Journal of Control, vol. 89, no. 9, pp. 17771787, 2016.

[25] M. Basin, Y. Shtessel, and F. Aldukali, "Continuous finiteand fixed-time high-order regulators," Journal of the Franklin Institute. Engineering and Applied Mathematics, vol. 353, no. 18, pp. 5001-5012, 2016.

[26] A. Polyakov, D. Efimov, and W. Perruquetti, "Robust stabilization of MIMO systems in finite/fixed time," International Journal of Robust and Nonlinear Control, vol. 26, no. 1, pp. 6990, 2016. 
[27] A. Levant, "Higher-order sliding modes, differentiation and output-feedback control," International Journal of Control, vol. 76, no. 9-10, pp. 924-941, 2003.

[28] M. T. Angulo, J. A. Moreno, and L. Fridman, "Robust exact uniformly convergent arbitrary order differentiator," Automatica. A Journal of IFAC, the International Federation of Automatic Control, vol. 49, no. 8, pp. 2489-2495, 2013.

[29] A. Polyakov and L. Fridman, "Stability notions and Lyapunov functions for sliding mode control systems," Journal of the Franklin Institute. Engineering and Applied Mathematics, vol. 351, no. 4, pp. 1831-1865, 2014.

[30] E. Bechkenback and R. Bell Man, Inequalities, Springer, Berlin, Germany, 1971.

[31] J. J. E. Slotine and W. Li, Applied Nonlinear Control, PrenticeHall, Englewood Cliffs, NJ, USA, 1991.

[32] H. K. Khalil, Nonlinear Systems, Prentice-Hall, Upsaddle River, NJ, USA, thrd edition, 2002. 


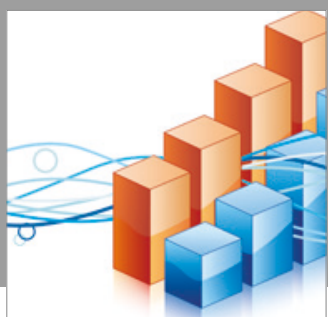

Advances in

Operations Research

vatersals

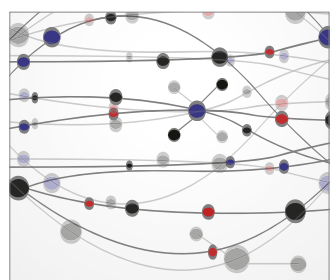

\section{The Scientific} World Journal
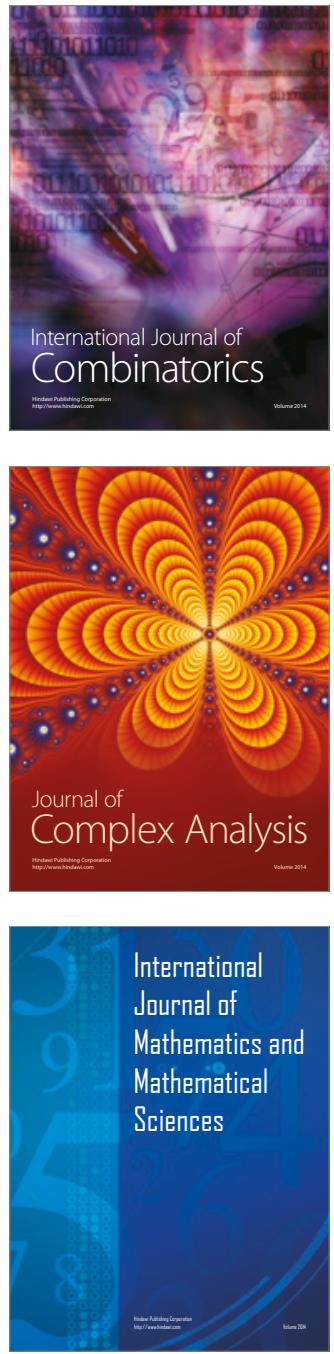
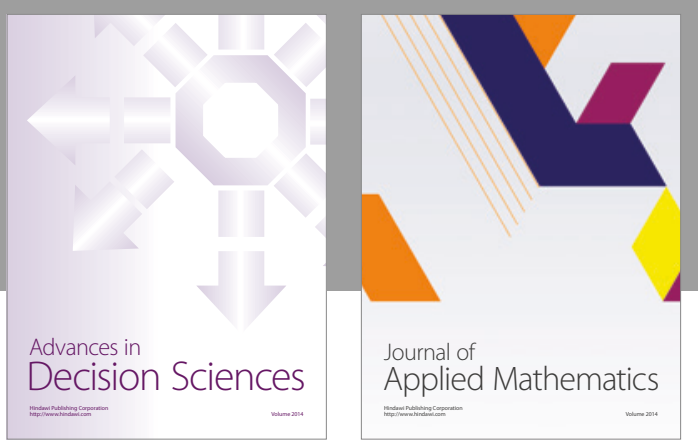

Algebra

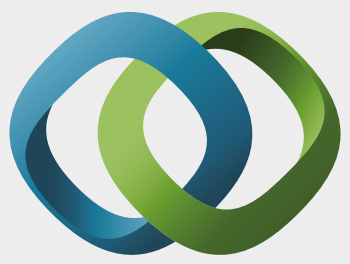

\section{Hindawi}

Submit your manuscripts at

https://www.hindawi.com
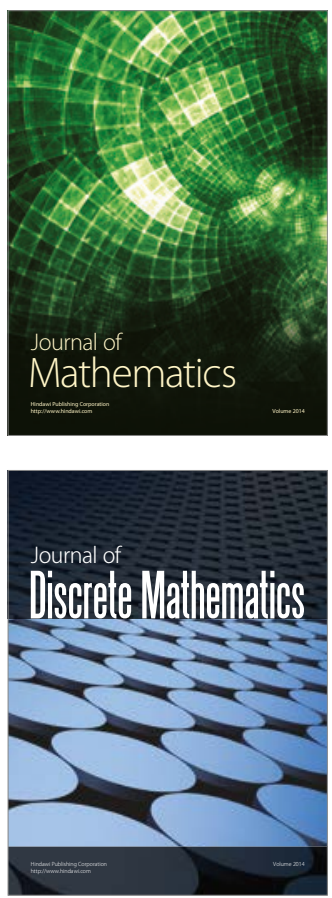

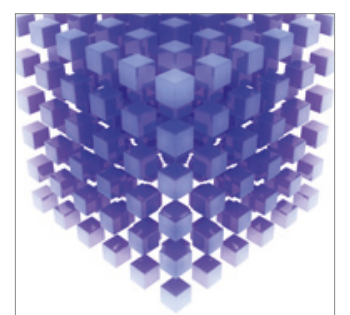

Mathematical Problems in Engineering
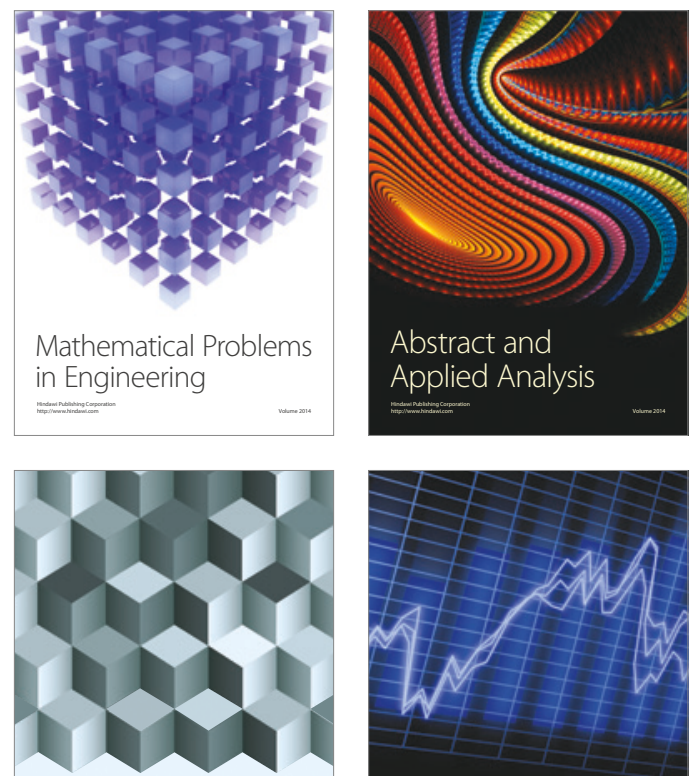

Journal of

Function Spaces

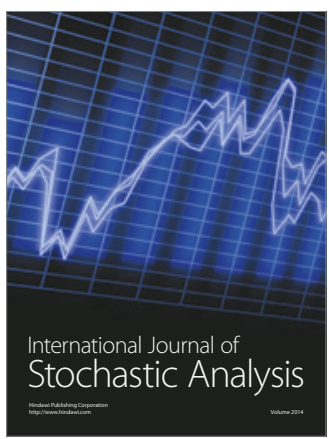

Probability and Statistics
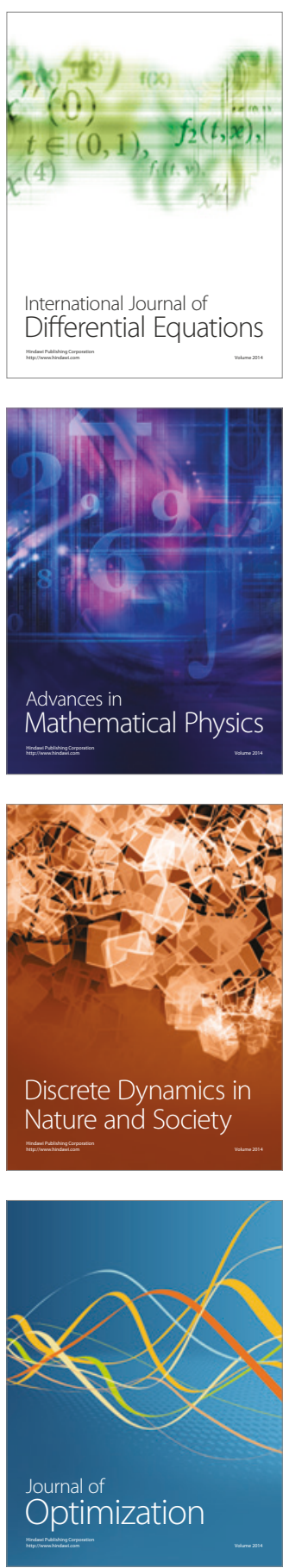